\title{
Representaciones sociales sobre la violencia recibida y ejercida en los noviazgos juveniles heterosexuales en el Valle de Aburrá - Colombia*
}

Social representations of violence received and exercised in heterosexual youth courtships in the Valley of Aburrá - Colombia

Representações sociais da violência recebidos e exercidas em namoros jovens heterossexuais no Vale da Aburrá - Colômbia

\author{
César Augusto Jaramillo Jaramillo** \\ Henry Holguín Osorio ${ }^{* * * *}$ \\ Liliana Patricia Ramírez Ramírez
}

\section{Resumen}

El artículo presenta resultados parciales de la investigación realizada según un diseño metodológico mixto, que relaciona estrategias de investigación cualitativas y cuantitativas, pero del cual se mostrará aquí solo los hallazgos cuantitativos que se lograron captar desde la racionalidad exploratoria-descriptiva, con la que se identificaron algunas representaciones sociales sobre la violencia recibida y ejercida en los noviazgos juveniles heterosexuales en el Valle de Aburrá. En este ejer-

Resultado de la Investigación -Algunas representaciones sociales sobre la violencia en los noviazgos juveniles heterosexuales en el Valle de Aburrá - Colombia. Investigadores: César Augusto Jaramillo Jaramillo - Institución Universitaria de Envigado, Henry Holguín Osorio - Fundación Universitaria Luis Amigó - Sede Medellín y Liliana Patricia Ramírez Ramírez - Universidad Coo perativa de Colombia - Sede Medellín. Realizado en el 2015.

** Psicólogo, Magister, Especialista, Investigador y Docente universitario de la Institución Universitaria de Envigado, ceauja@gmail.com

** Psicólogo, Magister, Especialista, Investigador y Docente universitario de la Fundación Universitaria Luis Amigó - Sede Medellín, henryholguínosorio@gmail.com

**** Psicóloga, Magíster, Especialista, Estudiante de Doctorado, Investigadora y Docente universitario de la Universidad Cooperativa de Colombia - Sede Medellín, lilitaram@gmail.com 
cicio investigativo se logró captar cómo perciben las y los jóvenes sus relaciones amorosas y cómo las tiñen con violencia psicológica y física, engrosando así las estadísticas públicas de los sistemas de vigilancia epidemiológica estatal de los contextos de los municipios antioqueños (Colombia) a los que pertenecen y en los que fueron estudiados.

Palabras claves: Representaciones sociales, noviazgos heterosexuales, juventud, violencia recibida y ejercida.

\section{Abstract}

The article presents results of research conducted by a mixed methodological design, linking strategies of qualitative and quantitative research, but which will be displayed here only quantitative findings that managed to capture from the exploratory - descriptive rationality with which identified some social representations received and exercised violence in heterosexual youth courtships in the Valley of Aburrá. In this research exercise it was able to capture how they perceive and young love relationships and how stained with psychological and physical violence, thus swelling the public statistics of the state epidemiological surveillance systems in the context of the municipalities of Antioquia (Colombia) to the they belong and in which were studied.

Keywords: Social representations, heterosexual dating relationships, youth, received and exercised violence.

\section{Resumo}

$\mathrm{O}$ artigo apresenta resultados de pesquisa realizada por uma concepção metodológica mista, coordenando as estratégias de pesquisa qualitativa e quantitativa, mas que será exibido aqui apenas resultados quantitativos que conseguiram capturar a partir da racionalidade exploratório-descritivo com o qual identificou alguns representações so- 
ciais recebidas e exercido violência em namoros jovens heterossexuais no Vale do Aburrá. Neste exercício, a pesquisa foi capaz de captar a maneira como percebem e os relacionamentos novos do amor e como manchada com violência psicológica e física, engrossando as estatísticas públicas dos sistemas de vigilância epidemiológica do Estado no contexto dos municípios de Antioquia (Colômbia) para o eles pertencem e em que foram estudados.

Palavras-chave: Representações sociais, heterossexuais relações de namoro, jovens, violência recebido e exercido.

\section{Introducción}

En el contexto colombiano, así como en la mayoría de los países occidentales, se conocen y divulgan estudios en el medio científico que muestran una prevalencia cada vez mayor de la violencia en las relaciones afectivas entre los jóvenes. Entre estos se encuentran estudios como los divulgados por la Organización panamericana de la salud y la Organización mundial de la salud (Ops-Oms, 2011), Programa integral sobre las violencias de género (2010), el estudio sobre tolerancia social e institucional a la violencia basada en género en Colombia realizado por Ramírez y Núñez (2010), las investigaciones de Rey-Anacona (2008-2009), Rey-Anacona, Mateus-Cubides, y Bayona-Arévalo (2010), Rivera, Allen, Rodríguez, Chávez y Lazcano (2007), Caycedo, Cubides, Martín, Cortés, Berman, Oviedo y Suárez (2007), Botero, Herrera, Reyes y Urrea (2006), entre otros.

Investigaciones relacionadas con el tema de violencias en parejas de jóvenes, apuntan a establecer este problema como uno de los riesgos más importantes para su desarrollo, puesto que se encuentra en una etapa en la que se construyen sus relaciones afectivas entre pares.

En la juventud las relaciones interpersonales se dan en el marco de un proceso de socialización, configuración de la identidad y la confianza, a través del cual, él y la joven exploran nuevas experiencias, se 
apropian de los roles de género y asumen nuevos modos de vida; este tránsito es alimentado por la influencia de los pares, los modos de relación, la familia y las prácticas y creencias del contexto sociocultural, entre otros; todo esto aporta al joven referentes para la construcción de su realidad.

En este orden de ideas, González y Santana (2001a, 2001b, como se cita en Urbiola 2014, p.151) plantean que la anterior sumatoria de condiciones puede contribuir a que sea una etapa de la vida vulnerable a tolerar determinadas relaciones abusivas o a que se construya una relación asfixiante.

Según los datos del Instituto Nacional de Medicina Legal (2008, p.25, citado en Ariza, 2009, pp. 104-105), en el 2007 en Colombia, se presentaron 46315 víctimas de violencia en las relaciones de pareja. De los 40659 casos desagregados por sexo, 36704 eran mujeres. En otros datos presentados por agencias de la Organización de Naciones Unidas, tales como el Fondo de Desarrollo de las Naciones Unidas para la Mujer-Unifem, Fondo de Población de las Naciones Unidas-Unfpa y la Organización Internacional para las Migraciones-Oim, sostienen con respecto a la edad en la que se comienza el maltrato de género o de pareja, que el 18 por ciento del total de 1080 personas en diez ciudades de las cinco regiones de Colombia entre 11 y 17 años, reconoció haber recibido alguna agresión (Nullvalue, 2010).

En Antioquia, la Dirección Seccional de Salud y Protección Social, registra en el Portal de la Dirección Seccional, “opción Sistema de vigilancia”, los eventos objeto de vigilancia epidemiológica en salud mental, tales como: 2526 casos de violencia física, 718 casos de abuso sexual y 601 casos de violencia psicológica reportados por el Sistema Vigilancia Epidemiológica en Salud Mental en Antioquia- Sivigila (2010), para un total de 3845 eventos registrados en el 2010. En el 2011 se registran 4 328 casos de violencia física, 1430 casos de abuso sexual y 1537 casos de violencia psicológica Sivigila (2011), para un total de 7 295, lo cual muestra una escalada significativa de la problemática, prácticamente duplicándose entre el año 2010 y el 2011 y presentándose 3450 casos nuevos en el año 2011, ello sin contar con los datos consolidados del 
2012 y con la debilidad de los sub-registros por los casos no reportados por la comunidad (Gobernación de Antioquia, 2013).

En el panorama internacional y según la Organización Mundial de la Salud, 3 de cada 10 adolescentes denuncian que sufren violencia en el noviazgo, lo cual invita a reflexionar sobre los comportamientos que tienen actualmente los jóvenes en sus primeras relaciones afectivas que se constituyen en las inaugurales formas de simbolizar y actuar con la pareja (Ops-Oms, 2011).

Con Sugarman y Hotaling (1989) se puede definir la violencia en la relación romántica, como la perpetración o amenaza de un acto de violencia por parte de por lo menos un miembro de una pareja no casada, sobre el otro miembro en el contexto de una relación romántica o de cortejo.

Algunos investigadores, han estimado que la violencia en la pareja adolescente comienza a presentarse entre los 15 y los 19 años, y lo más grave es que se ha interpretado como un acto de amor en una mayoría significativa de los casos por el agresor o el agredido (Poo y Vizgarra, 2008).

Otros autores, como Duschatzky y Corea (2009), centran sus estudios en el aprendizaje de estas violencias en contextos sociales los cuales son replicados, perpetuados y padecidos por las y los jóvenes en sus relaciones amorosas en calidad de víctimas y victimarios, tal como lo planteó Hanson (2002), quien identificó que:

Aproximadamente el 20\% de los adolescentes han experimentado alguna forma de violencia en el noviazgo (Bergman, 1992; O’Keefe, Brockopp \& Chew, 1986), expresada a través de comportamiento físicamente agresivo y de violencia psicológica. Este porcentaje de jóvenes ha sido objeto de agresión y coerción sexual que incluía besos no deseados, abrazos, contacto genital y en algunos casos relación coital (citado en Duschatzky y Corea, 2009, p.147).

Otras investigaciones internacionales señalan que las cifras de prevalencia de violencia en las relaciones de noviazgo entre jóvenes, fluctúa entre el 9 y el 46\%; esta variación se relaciona con la falta de consenso 
respecto de la definición de violencia, con el tipo de instrumentos de recolección de datos usados, con el período de tiempo reportado (violencia en el último año o violencia a lo largo de la vida) y con el tipo de población estudiada (Price, Byers, Sears, Whelan \& Saint-Pierre, 2000; Glass, Freland, Campbell, Yonas, Sharp \& Kub, 2003; Amurrio, Larrinaga, Usategui y Del Valle, 2008).

Otros autores señalan cifras de $23 \%$ para la violencia grave y $51 \%$ si se consideran todas las formas de violencia (Fagot \& Browne, 1994, citado por Graves, Sechrist, Whiste \& Paradise, 2005). Así mismo mencionan que en estudiantes universitarios (White \& Koss, 1991) se reporta una incidencia de $37 \%$ de varones y $35 \%$ de mujeres que infligieron alguna forma de agresión física (Graves et al., 2005).

En Chile las cifras no difieren de las encontradas en otros países; estudios realizados con estudiantes universitarios, señalan que alrededor del 50\% de los encuestados refiere haber recibido agresión psicológica, y aproximadamente un cuarto reconoce haber recibido violencia física al menos una vez a lo largo de la vida (Poo \& Vizgarra, 2008, p.82).

Además, se considera que una consecuencia importante a largo plazo de la violencia en el noviazgo, sería la repetición de esta conducta en futuras relaciones (Glass, Freland, Campbell, Yonas, Sharp \& Kub, 2003, citados en Póo \& Vizcarra, 2008, p.82).

Los anteriores resultados demuestran que existen entre las parejas, formas de relacionarse que estimulan la agresión y la violencia en las relaciones afectivas de las y los jóvenes. Retomando lo anterior, el propósito central del presente estudio exploratorio-descriptivo, se orienta a recabar información pertinente sobre la violencia recibida y ejercida en los noviazgos juveniles heterosexuales en el Valle de Aburrá, en el Departamento de Antioquia-Colombia, dirigiéndose a identificar algunas las representaciones sociales sobre la violencia en el noviazgo heterosexual, en este caso en el contexto estudiado, como producto de la transmisión y el intercambio cultural que se da a través de las influencias externas que reciben cotidianamente los jóvenes en sus interaccio- 
nes, modos de socialización, de la información a la que acceden a través de los medios de comunicación, la internet, las nuevas tecnologías, las ideologías, de la propia época contemporánea, de la religión, del saber académico y las reflexiones que el jóven hace frente al conocimiento al que tiene acceso en sus interacciones cotidianas; todo esto incide directamente en sus percepciones, creencias, comportamientos y actitudes y por todas estas influencias se dan el tipo de representaciones sociales que se construyen, permanecen o mutan e incluso pueden desaparecer como puede pasar con todo fenómeno sociocultural y con el estudiado.

Así mismo genera interés que la violencia registrada en el noviazgo juvenil heterosexual, frecuentemente pase desapercibida en el ámbito social, en tanto se visibiliza más la violencia intrafamiliar, entre padres, hermanos o hijos (Ariza, 2013), otorgando poca importancia a lo que puede llegar a suceder con los jóvenes en sus primeras relaciones amorosas; además, no existe un marco legal claro para el abordaje de la problemática, ni se registra de manera sistemática en los sistemas de información de salud pública, en los cuales abunda el subregistro.

Dada la magnitud de la gravedad de este problema en el Valle de Aburrá, en Antioquia y Colombia, interesa interrogar ¿Cómo los jóvenes se representan socialmente las violencias en sus noviazgos? para comprender los sentidos asociados a este tipo de interacciones, especialmente las que se ligan con la violencia en el noviazgo.

Cabe mencionar aquí que para Jodelet (1985) las representaciones sociales son una forma de conocimiento social que orienta a los sujetos en su manera de interpretar y de pensar la realidad cotidiana. Se relaciona con la actividad mental desplegada por individuos y grupos a fin de fijar su posición en relación con situaciones, acontecimientos, objetos y comunicaciones que les conciernen.

Finalmente, es importante clarificar que el concepto representaciones sociales designa una forma de conocimiento específico, el saber del sentido común, cuyos contenidos manifiestan la operación de procesos generativos y funcionales socialmente caracterizados. En sentido más amplio, designa una forma de pensamiento social (Moscovici, 1985; Araya, 2002). 


\section{Metodología}

La investigación que sirvió de marco a este artículo se desarrolló desde un paradigma de investigación mixta, que relaciona estrategias de investigación cualitativas (Galeano, 2009) y cuantitativas (Hernández, Fernández y Baptista, 2006). El componente cuantitativo se desarrolló a partir de un estudio exploratorio-descriptivo y el cualitativo se realizó desde el método etnográfico y, ambos combinados, permitieron a los investigadores indagar y caracterizar las representaciones sociales sobre la violencia en los noviazgos juveniles heterosexuales presentes en las y los participantes de los municipios del Valle de Aburrá que se investigaron.

Las representaciones sociales que se identificaron en el componente cualitativo de la investigación mixta fueron: las percepciones, las creencias, las actitudes y los contextos de producción de las representaciones sociales, se observaron tal y como se dieron en su escenario natural para después ser analizados (aquí no se exponen dichos hallazgos).

Desde la perspectiva cuantitativa se cuantificaron y analizaron algunos aspectos sobre la violencia en los noviazgos juveniles heterosexuales en los municipios del Valle de Aburrá, teniendo en cuenta la violencia ejercida y la violencia recibida por parte de las y los jóvenes.

Para lograr tal propósito, se aplicó una metodología de investigación de carácter exploratorio-descriptivo, para generar una mayor aproximación y familiarización con las representaciones sociales sobre la violencia en los noviazgos juveniles, se buscó identificar tendencias, relaciones potenciales entre variables y establecer el "tono" de posteriores investigaciones e intervenciones, siguiendo lo planteado por Hernández, Fernández y Baptista (2006).

El tipo de información recopilada fue determinada por los investigadores, con la participación de 413 actores juveniles pertenecientes a nueve (9) municipios que se reseñan a continuación: Medellín, Sabaneta, la Estrella, Caldas, Bello, Girardota, Itagüí, Envigado y Barbosa. Esta fue la comunidad estudiada a través de la realización de 13 talleres en los cuales la población objeto se abordó en nueve (9) instituciones educativas y tres (3) instituciones de educación superior, a través de 
metodologías interactivas, instrumentos y conversatorios, que permitió la profundizaron desde la propia visión juvenil sobre la problemática estudiada.

Es pertinente clarificar que la información de este artículo relaciona solo los datos cuantitativos, que se procesaron mediante software y análisis de información cuantitativa, con ayuda del SPSS (Statistical Packageforthe Social Sciences) versión 22 (Licencia adquirida por la Fundación Universitaria Luis Amigo). Cabe anotar aquí, que los datos y los hallazgos se analizaron independientemente para generar resultados, discusiones, conclusiones y recomendaciones para cada racionalidad metodológica empleada, pero aquí solo se presentan los análisis y la discusión cuantitativa.

\section{Instrumento para la recolección de información: Cuestionario V.E.R.A.}

Este es un instrumento de tipo autoinforme y de fácil aplicación, diseñado y validado en Colombia por Urbiola (2014). Está compuesto por tres partes, la primera es la percepción de la violencia recibida por el informante en sus relaciones de pareja; la segunda, es la percepción de la violencia ejercida por los participantes contra sus parejas; y la tercera, la percepción de qué tan violentas son estas conductas que se han señalado como recibidas o ejercidas; este último aspecto del instrumento no se consideró para la recolección y el análisis de la información que requirió este estudio.

El instrumento tal como lo describe Urbiola (2014), consta de 41 situaciones de violencia, de diferentes grados, que el informante debe señalar si las ha recibido, las ha ejercido y si le parecen violentas. Para informar sobre la violencia ejercida o recibida, el informante dispone de una escala Likert de 6 posibilidades de respuesta (o = nunca, $1=$ una vez, $2=$ de 2 a 5 veces, $3=$ de 6 a 10 veces, $4=$ de 11 a 15 veces y 5 $=$ más de 15 veces). Por su parte, la percepción de violencia dispone de 5 alternativas de respuesta: no es violencia, poco violento, algo violento, bastante violento y muy violento. Como requisito los participantes debían de haber mantenido como mínimo una relación superior a un mes (Rodríguez Sánchez y González, 2006; Wolfe, Scott, Reitzel-Jaffe, Wekerle, Grasley \& Straatman, 2001). 
El cuestionario consta de cinco (5) subescalas: violencia física, violencia sexual, violencia psicológica social, violencia psicológica humillación y violencia psicológica celos (Urbiola, 2014).

Cabe señalar que los ítems del cuestionario Vera, clasifican los tipos de violencia a partir del método Delphi, que proporciona un procedimiento estructurado de toma de decisiones para la elaboración del instrumento, comenzando en una serie de fases de Mohedano (2008, citado en Urbiola, 2014, p.129).

Cuadro 1. Dimensiones de la violencia según Urbiola (2014)

\begin{tabular}{|c|c|c|}
\hline DIMENSIONES & DESCRIPCIÓN & INSTRUMENTO \\
\hline $\begin{array}{l}\text { Percepción de la } \\
\text { violencia física } \\
\text { ejercida } \\
\text { Percepción de la } \\
\text { violencia física } \\
\text { recibida }\end{array}$ & $\begin{array}{l}\text { Violencia Física: Hará referencia a } \\
\text { cualquier acto no accidental que } \\
\text { Provoque o pueda provocar daño en el } \\
\text { cuerpo. Se incluirían aquí acciones } \\
\text { tales como patear, morder, golpear con } \\
\text { puños u objetos, dar palizas, emplear } \\
\text { un arma, abofetear, empujar, arrojar } \\
\text { objetos, estrangular, escupir, etc. }\end{array}$ & \\
\hline $\begin{array}{l}\text { Percepción de la } \\
\text { violencia Sexual } \\
\text { ejercida } \\
\text { Percepción de la } \\
\text { violencia Sexual } \\
\text { recibida }\end{array}$ & $\begin{array}{l}\text { Violencia Sexual: Se presenta } \\
\text { cuando se impone un contacto y una } \\
\text { cierta práctica sexual contra la volun- } \\
\text { tad de la persona víctima (tocamien- } \\
\text { tos, abusos, agresiones, inducción a la } \\
\text { prostitución, etc.). }\end{array}$ & $\begin{array}{l}\text { Cuestionario } \\
\text { (Validado en } \\
\text { Colombia por } \\
\text { Urbiola, 2014). }\end{array}$ \\
\hline $\begin{array}{l}\text { Percepción de la } \\
\text { violencia Psicológi- } \\
\text { ca-Social ejercida } \\
\text { Percepción de la } \\
\text { violencia Psicológi- } \\
\text { ca-Social recibida }\end{array}$ & $\begin{array}{l}\text { Violencia Psicológica-Social: } \\
\text { Comprende aquellos actos intencio- } \\
\text { nados que producen desvalorización o } \\
\text { sufrimiento en las víctimas. Se } \\
\text { diferenciaron diversos tipos principa- } \\
\text { les de maltrato emocional o psicológi- } \\
\text { co, los más relevantes para la creación } \\
\text { de este cuestionario son: }\end{array}$ & \\
\hline
\end{tabular}


Humillación: recoge conductas de ridiculización, humillación, amenazas verbales e insultos, que la persona agresora comete contra la víctima. Las consecuencias de esta Violencia Psicológica son baja autoestima, inseguridad, sentimientos de incapacidad, impotencia y la creencia de que no se podrá desarrollar ninguna actuación válida.

Aislamiento social: hace referencia a las conductas dirigidas a impedir o dificultar el contacto con otras personas para evitar así que se puedan tener otros criterios, comparar su situación con la de otras personas, o pedir y recibir ayuda.

- Celos y posesividad: abarca las conductas tendentes a polarizar la atención de la persona víctima $\mathrm{y}$, gracias a ello, impedir que pueda dedicarse a otras actividades o personas.

Fuente: Urbiola (2014)

\section{Descripción de las y los jóvenes participantes}

Para contextualizar los hallazgos de la investigación, se partió de una descripción de las (245-60\%) mujeres y los (166-40\%) hombres de los 413 jóvenes participantes en el estudio, en términos de su género por cada tres (3) participantes de género femenino se contó con dos (2) participantes de género masculino, el rango de edad fue definido entre los 14 y los 28 años (Congreso de Colombia, 2013), las edades de mayor frecuencia esta con el 65\% de los participantes están entre los 14 y 17 años, es importante clarificar que de cada 5 jóvenes participantes 3 pertenecían a este rango de edad, mientras el grupo de edades menos representativo estuvo entre los 25 y 28 años, con un 3,1 \% de participación.

En cuanto al estado civil, el comportamiento de esta variable fue el esperado en una población mayoritariamente adolescente y vinculada en su mayoría a instituciones educativas de enseñanza media vocacional, el $87,7 \%$ responde que es soltero, pero llama la atención que el 
8,39 \% se encuentre en unión libre; sin embargo, no es extraño pues en Colombia este tipo de unión en las últimas décadas del Siglo xxi es recurrente no solo en las poblaciones adolescentes, si no también jóvenes y adultas.

En torno al nivel educativo el grueso de los sujetos de la investigación, correspondiente al 76,7\% cursa estudios secundarios, seguidos de los Universitarios con un 12,2\%; también se destacan el 7,19\% que posee estudios técnicos, esto es congruente con la población objeto que fue seleccionada.

La ocupación se destacó la de estudiante con un 94,2\% se presenta en la mayor parte de la población participante, llama la atención que las cifras que le siguen sean la de trabajador informal y de empleado con un 4\%, lo cual demuestra como para muchos jóvenes es necesaria su articulación con el sistema productivo, para poder desarrollar su proyecto de vida personal.

Con respecto del estrato socioeconómico, los estratos $3(46,2 \%)$ y 2 (37,1\%), son los más representativos, seguidos del 1, y luego del 4 y 5. Este rasgo corresponde con el tipo de instituciones educativas que fueron parte de la investigación que en su mayoría son públicas.

La tipología familiar preponderante en los participantes es la nuclear, representada en un 61,1\% de las respuestas, esto significa que 3 de cada 5 participantes provenía de este tipo de familia, seguida de la monoparental femenina con un $19,42 \%$, representada en uno de cada cinco participantes. La siguiente en orden de importancia fue la extensa con $8,63 \%$ de las respuestas. Vale la pena anotar que estas tres tipologías de familia son las más comunes en recurrencia en el contexto colombiano.

Cabe señalar que estas preguntas fueron cerradas, y hacían parte del instrumento V.E.R.A. aplicado (cuantitativo), y algunas de las preguntas se pudieron contrastar con las manifestaciones discursivas y las prácticas asociadas a las representaciones sociales sobre la violencia en el noviazgo juvenil heterosexual, emergentes en los talleres. 


\section{Hallazgos y discusión}

En las siguientes líneas se muestra el análisis realizado a partir del cuestionario V.E.R.A., ${ }^{1}$ con la presentación y discusión de los resultados, es importante aclarar que se acudirá a los tipos de violencia descritos por Bosch \& Ferrer (2003) y Follingstad, Rutledge, Berg et al. (1990, citado en Urbiola, 2014), estos son: violencia física, violencia sexual, violencia psicológica social, violencia psicológica humillación y violencia psicológica celos (control) (Urbiola, 2014).

En desarrollo de las anteriores categorías los jóvenes participantes respondieron en el cuestionario de violencia ejercida y recibida en el noviazgo, con qué frecuencia han vivido estas experiencias. Para ello se tuvo en cuenta, las relaciones que los jóvenes han conservado o en las que han permanecido más de un mes.

Para la discusión de los datos cuantitativos se parte de una clasificación de la violencia en el noviazgo en: baja entre $1 \%$ y $15 \%$, media entre $15,1 \%$ y $30 \%$ y alta 30,1\% y más, ello basado en los promedios presentados en las investigaciones que sirvieron como marco referencial y antecedentes para el presente ejercicio de análisis cuantitativo (p.j.: Escoto, González, Muñoz y Salomon, 2007; Echeburúa, Fernández y Corral, 2008).

\section{Violencia física}

En esta primera categoría se da una aproximación sobre las acciones de violencia física indagadas, frente a las cuales, las y los participantes reconocen que se pueden presentar dentro de la relación de pareja de manera significativa y registraron en orden de severidad las siguientes: ser empujado/empujar; recibir mordiscos o tirones de pelo/dar mordiscos o tirones de pelo; ser pellizcado/pellizcar; recibir bofetadas o golpes/dar bofetadas o golpes; y recibir patadas/dar patadas en sus relaciones de noviazgo juvenil.

1 Cuestionario de violencia ejercida y recibida en jóvenes y adolescentes. V.E.R.A. (Urbiola, 2014, p.128). 
De los datos se permite concluir que cuatro (4 - 39\%) de cada 10 jóvenes participantes han sido empujados por su pareja y que entre cuatro y cinco (45\%) reconocen que han empujado a propósito a su pareja entre una y más de quince veces.

Entre 2 y 3 (25,7\%) de cada 10 de los participantes de género masculino o femenino han recibido un mordisco o un tirón de pelo en por lo menos en una ocasión y dos $(2-22 \%)$ de cada 10 participantes han dado mordiscos o tirones de pelo por lo menos en más de una vez y hasta más de quince veces en su relación de noviazgo.

Por lo menos dos $(2-23 \%)$ de cada 10 de los participantes de género masculino o femenino han sido pellizcados con intención de daño por su pareja y que dos $(2-19,2 \%)$ de cada 10 participantes mujer u hombre han dado pellizcos a su novio o novia por lo menos una vez y hasta más de quince veces en el contexto de su relación de noviazgo.

Por lo menos uno (1 - 12,8\%) de cada 10 de los participantes de género masculino o femenino ha pegado bofetadas o golpes, y uno (1 12,6\%) de cada 10 participantes (mujer u hombre) pegó bofetadas o golpes por lo menos una vez y hasta más de quince veces en el contexto del noviazgo juvenil.

De estos datos se deduce también que por lo menos uno (1 - 10,4\%) de cada 10 de los participantes de género femenino o masculino ha pegado una patada, y uno (1 - 9,7\%) de cada 10 participantes de ambos géneros ha dado patadas por lo menos entre una a quince veces en el contexto de su relación de noviazgo.

Los jóvenes de acuerdo a las experiencias vividas expresaron que existen actos que parecieran ser simples, $\mathrm{y}$, sin embargo, son agresivos e intencionales. Manifiestan que tanto para quien recibe o para quien agrede, pasan inadvertidos estos actos como violentos, estos parecen ser parte de la relación y por ello no se nombran por ser experiencias comunes y corrientes que se viven en las relaciones de pareja, cabe mencionarse aquí que aunque no sean percibidos como violencia lo son y afectan profundamente los sujetos implicados. 
En esta primera categoría se puede evidenciar que una gran mayoría de los jóvenes que viven cotidianamente sus relaciones de noviazgos heterosexuales, reconocen que hay violencia física, y en el diario vivir se ha naturalizado puesto que bajo el modelo machista arraigado de la cultura de los nacidos en el Valle de Aburrá en medio de la denominada cultura paisa, que hace que el hombre ejerza mayor nivel de autoridad sobre las mujeres y los hijos en el núcleo familiar y en la relación de pareja ejerza una dominación androcéntrica, que en los casos particulares estudiados también se perciba y evidencie, pues existen jóvenes de género masculino que en el contexto estudiado no escapan de esta impronta cultural de macho paisa activo, de lo público y dominador y que las mujeres paisas representen el rol pasivo, de lo privado y la dominada (Jaramillo, Holguín y Ramírez, 2015).

Se puede describir que los jóvenes reconocen que estos actos están ligados a la violencia, y logran diferenciar si existe o no agresión física en sus relaciones de pareja, sin embargo, cuando ellas suceden en muchas ocasiones no las nombran o pasan a silenciarlas (Jaramillo, Holguín y Ramírez, 2015).

El reconocimiento de la violencia en el noviazgo juvenil entre los mismos jóvenes es muy bajo y se da de manera recurrente, según Peña, Zamorano, Hernández, Hernández, Vargas, y Parra (2013) expresan que una investigación realizada en Estados Unidos en el año 2003 por los centros para la prevención y el control de la enfermedad (Centers for Disease Control and Prevention, 2013) hallaron en una muestra de 15 ooo realizada con jóvenes de secundaria pertenecientes a instituciones públicas y privadas de 50 estados y el distrito de Columbia, se halló que el $8,9 \%$ de los varones y el $8,8 \%$ de las mujeres registraron haber sido objeto de violencia física por parte de su pareja.

Si se compara este dato con el obtenido en el ejercicio llevado aquí en el Valle de Aburrá-Colombia, se podría afirmar categóricamente que lo encontrado aquí dispara esta cifra anteriormente mostrada, pero si se mira el contraste con otros datos internacionales se tiene que las cifras de prevalencia de violencia en el noviazgo juvenil fluctúan entre 
el 9\% y el 46\%; esta variación se relaciona con la falta de consenso respecto de la definición de violencia, con el tipo de instrumentos de recolección de datos usados (cuantitativos y cualitativos), con el período de tiempo reportado, entre otras variables, p.j., lo contextual.

Otros autores señalan cifras de $23 \%$ para la violencia grave y $51 \%$ si se consideran todas las formas de violencia (Graves, Sechrist, Whiste \& Paradise, 2005; Fagot \& Browne, 1994, citados por Graves et al., 2005), si se toma esta cifra en consideración para el presente análisis y si se promedian los cinco (5) tipos de violencia física que se analizaron con el cuestionario V.E.R.A., se tiene en el presente estudio que el 22,1\% de las y los jóvenes fue objeto de algún tipo de violencia física y que el $21,7 \%$ violentó físicamente a su pareja, lo que da un promedio de 21,9\% para cualquier tipo de violencia física recibida o aplicada en la relación de noviazgo juvenil, esta cifra ubica la violencia física hallada en la violencia media, basada esta afirmación en lo que señalan los autores arriba nombrados.

Datos que vale la pena mencionar aquí, es que en estudiantes universitarios White \& Koss (1991, citados por Graves et al., 2005), reportaron una incidencia de $37 \%$ de varones y $35 \%$ de mujeres que infligieron alguna forma de agresión física, este dato sirve también como referente en la medida que el estudio aquí hecho también abordó población universitaria $(12,2 \%)$ que también reportaron cinco formas de violencia física, pero de manera afortunada la cifra se aleja de la del estudio citado, pues los datos rondan por el $22 \%$ de violencia física recibida y ejercida, lo que hace presumir que la violencia física hallada en el noviazgo juvenil universitario del Valle de Aburrá se reduce en un $15 \%$ comparada con la cifra citada y se podría calificar, a pesar de ello, con tendencia a media.

\section{Violencia Sexual}

Inicialmente en esta segunda categoría las formas de violencia sexual, en su orden de severidad, se presentan de la siguiente forma: ser 
obligado u obligar a besar; ser obligado u obligar a mantener relaciones sexuales como manera de demostrar el amor, intentaron o intentó mantener relaciones sexuales sin violencia física con su pareja; se le pidió no usar o ridiculizó a su pareja por el uso de anticonceptivos; fue obligado u obligó a tocar sexualmente a su pareja aunque no lo apeteciera y, finalmente, fue obligado u obligó a su pareja a mantener relaciones sexuales aunque no lo apeteciera.

Se infiere que por lo menos dos $(2-21,3 \%)$ de cada 10 de los participantes de género femenino y masculino reconocieron ser obligada/o por su pareja género femenino o masculino a besar aunque no lo deseara, por lo menos en más de una ocasión y que entre uno y dos (15,7\%) de cada 10 participantes manifestaron que intentaron a obligar a que su pareja lo besara, aunque no lo deseara, por lo menos una vez y hasta más de quince veces en el contexto de su relación de noviazgo.

Por lo menos dos (2 -19,6\%) de cada 10 de los participantes de género femenino y masculino reconocieron ser obligados por su pareja a mantener relaciones sexuales como manera de demostrar el amor a su novio/a por lo menos en más de una ocasión, y que entre uno $(1-11,4 \%)$ de cada 10 participantes manifestó que si presionó a su pareja sexualmente con el argumento de que era una prueba de amor, por lo menos una vez y hasta más de quince veces en su relación de noviazgo juvenil.

Se refleja según en V.E.R.A., además, que por lo menos dos (2 $18,9 \%$ ) de cada 10 de los participantes de género femenino y masculino, su pareja intentó mantener relaciones sexuales sin violencia física y que uno (1-10,2\%) de cada 10 participantes manifestó que ella o el intentó mantener relaciones sexuales sin violencia física por lo menos una vez y hasta más de 15 ocasiones en el contexto de su relación de noviazgo.

Por lo menos uno $(1-9,9 \%)$ de cada 10 de los participantes de género femenino o masculino, reconoció que su pareja le pidió no usar o lo ridiculizó por el uso de anticonceptivos y él o ella le pidió o ridiculizó por el uso de anticonceptivos a su pareja, lo anterior en uno $(1-7 \%)$ de cada 10 participantes, por lo menos una vez en el contexto de su relación de pareja y hasta más de quince veces. 
Los datos muestran, así mismo, que por lo menos uno (1 - 8,7\%) de cada 10 de los participantes de género masculino o femenino fue obligado a tocar sexualmente aunque no lo apeteciera y lo ha hecho en uno $(1-6,5 \%)$ de cada 10 participantes, por lo menos una vez en el contexto de su relación de pareja.

En uno (1 - 9\%) de cada 10 jóvenes se reconoce que fue obligado por su pareja a mantener relaciones sexuales aunque no lo apeteciera y que menos de uno $(1-5,3 \%)$ reconoció que presionó a su pareja para obligarlo a tener relaciones sexuales aunque no quería.

Es pertinente mencionar que para las y los jóvenes en las etapas previas de enamoramiento y en la construcción de sus relaciones afectivas, es importante y necesario hablar y ejercer la sexualidad. También es común en estos, que se presenten dudas y desconocimientos, de cómo abordar estas realidades de la sexualidad recreativa y procreativa, para ellos y ellas estos tópicos se vuelven relevantes, pues dentro del noviazgo lo sexual está presente.

Cabe notar, que aunque estas frecuencias son bajas, es importante revisarlas y comprenderlas en los contextos reales de los noviazgos juveniles en las que se presentaron los casos, en el que apareció el registro de haber intentado, puesto que la violencia en los espacios de intimidad y la toma de decisión frente en el lugar de deseo sexual de la pareja, si no son tomados en cuenta generan estragos en las relaciones de noviazgo.

En los rastreos realizados para los antecedentes aparece otro análisis realizado por Sears, Byers y Price (2007, citado por Peña et al., 2013), quienes examinaron la "coocurrencia de la violencia física, psicológica y sexual y factores asociados y encontraron que un $43 \%$ de los adolescentes y el $51 \%$ de las adolescentes reportaron haber ejercido alguna conducta de maltrato físico, psicológico o sexual hacia su pareja” (p.29). Igualmente, en esta investigación se evidenció que "más mujeres que varones reportaron ejercer actos de violencia psicológica y física, mientras más varones reportaron ejercer actos de violencia sexual en sus relaciones de noviazgo" (p.29). 
Si se toma como referente el estudio anterior y lo comparamos con las cifras expresadas en el del Valle de Aburrá por las y los jóvenes, se ve con claridad en el presente estudio que la violencia sexual recibida promedia en un $14,5 \%$ y la ejercida en un $9,3 \%$, y que el promedio de estas dos llega a 11,9\%, lo cual permite afirmar que respecto a este estudio la violencia es 3 veces menor y podría calificarse con tendencia a baja.

De lo anterior se puede señalar que parece que existe en las relaciones de noviazgo evaluadas respeto por el cuerpo y las decisiones del otro, o sea se respetan los límites y las decisiones de ambos en la pareja cuando se refieren a temas sexuales, aunque no sin excepciones de vulneración más psicológica y en menor grado física en los aspectos sexuales evaluados.

Aunque las cifras de violencia sexual no registran datos alarmantes para esta categoría, se puede mencionar que hay un importante número de jóvenes que en sus relaciones de noviazgo presentan este tipo de violencia, y es pertinente señalar que en estos datos aunque se revelan frecuencias bajas pero significativas en las experiencias de noviazgo, pues uno (1) de cada diez (10) jóvenes reconocen que han ejercido y han recibido alguno de las variantes de este tipo de violencia entre una vez y hasta 15 veces en sus relaciones de pareja.

De lo anterior se desprende que se deben hacer programas preventivos para reducir el impacto de la violencia sexual en esta etapa del psicodesarrollo (adolescencia y juventud) para que no progrese en las etapas subsiguientes y se instale sobre todo como violencia intrafamiliar.

\section{Violencia psicológica social}

La tercera categoría en el orden de presentación de este tipo de violencia presenta la siguiente lógica: quedarse sin amigos porque su pareja lo presionó / presionar a su pareja y ésta se quedó sin amigos; su pareja insulta a sus amigo(a)s / la o él participante insulta lo(a)s amiga(o)s de su novio(a); su pareja no lo deja ver a los/as amigos/as / ella o él no dejan ver la(o) amiga(o) se su pareja; la pareja le impone con 
quien debe salir / la o el participante imponen con quien debe salir a su pareja y, cerrado esta categoría, su pareja insulta a su familia / ella o el insultan la familia de su novio(a).

En esta categoría, cuatro $(4-36,6 \%)$ de cada 10 de las y los jóvenes manifestaron haberse quedado sin amigos porque su pareja lo presionó para ello y dos $(2-22 \%)$ de cada 10 reconoció que presionó a su pareja para que no tuviese o se relacionase con amigos y se quedó sin ellos por esta causa, por lo menos en una y hasta más de quince veces.

Entre 3 y $4(34,4 \%)$ jóvenes de cada 10 participantes reconocieron que su pareja insulta a sus amigo(a)s y que tres (3-30\%) de cada 10 hace lo mismo con su pareja, respecto de los amigo(a)s de su novio o novia.

Tres (3 - 28,1\%) de cada 10 de las y los jóvenes manifestaron que su pareja no lo deja ver a los/as amigos/as y dos (2 - 18,6\%) de cada 10 participantes reconocieron que presionó a su pareja para que dejará de ver a sus amigos por lo menos en más de una vez y hasta más de quince veces.

Entre 2 y 3 (24,7\%) de cada 10 de las y los jóvenes manifestaron que su pareja le impone con quien debe salir y dos (2 -18,2\%) de cada 10 reconocieron que él o ella le imponen a su novio o novia con quien se debe salir por lo menos en más de una vez y hasta más de quince veces.

Uno (1-9.9\%) de cada 10 jóvenes participantes reconoce que su pareja insulta a su familia y que uno (1 -12,1\%) de cada 10 hace lo mismo con su pareja, respecto de la familia de su novio o novia.

En la subcategoría de la violencia psicológica social se evidenció que se ejerció algún tipo de violencia psicológica social sobre el $26,7 \%$ de los participantes y el 20,1\% ejerció, a su vez, sobre su pareja algún tipo de esta violencia, lo cual da como promedió un $23,4 \%$ de violencia psicológico-social, nombrándose como un índice de violencia media.

Aquí también es pertinente revisar si los jóvenes no identifican como violencia sus actos violentos, sean estos psicológicos o físicos, parece que no los reconocen como tales, lo cual sugiere que existe más este tipo violencia en los noviazgos que no fue revelada y que parece 
es poco nombrada. Al respecto Flores y Aguilar (1998) sustentan que algunas de las parejas juveniles en condición de noviazgo violento "no identifican el problema debido a que su convivencia no es tan frecuente como en los matrimonios, y las veces en que ocurre el maltrato es muy circunstancial, lo que impide dimensionar la magnitud del problema" (citados en Ramírez y Núñez, 2010, p.281).

\section{Violencia psicológica (humillación)}

En esta cuarta categoría propuesta por Urbiola (2014) los datos mostraron el siguiente orden de recurrencias de la más alta a la más baja: su pareja lo insulta cuando se enfada / la o él participante insultan a su pareja cuando se enfadan; su pareja le grita cuando se enfada / ella o él gritan a su pareja cuando se enfadan; su pareja le pone trampas para comprobar que le quiere / ella o él le pone trampas a su pareja para comprobar que él o ella le quiere; su pareja crítica los cambios en su forma de vestir o peinarse / la o él particípate critica de su pareja los cambios en su forma de vestir o peinarse; su pareja lo hace sentir que no vale para nada / ella o el hacen sentir a su pareja que no vale para nada; su pareja lo culpa de la violencia que se sufre en el noviazgo / ella o él culpan a su pareja de la violencia que se sufre en el noviazgo.

En este aspecto, cinco ( 5 - 49,6\%) jóvenes de cada 10 participantes reconocen que su pareja lo insulta cuando se enfada y que cuatro (4-40,9\%) de cada 10 hacen lo mismo con su novio o novia cuando se enfada.

Entre cuatro (4 - 44,1\%) jóvenes de cada 10 participantes reconocen que su pareja lo grita cuando se enfada y que cuatro $(4-39,5 \%)$ de cada 10 hacen lo mismo con su novio o novia cuando se enfada.

Tres (3 -33,2\%) jóvenes de cada 10 participantes reconocen que su pareja le pone trampas para comprobar que le quiere y entre 3 y 2 $(25,7 \%)$ de cada 10 participantes señalan que ponen a su novio o novia trampas para comprobar que le quiere. Dos $(2-22,3 \%)$ de cada 10 
participantes reconocen que su pareja critica los cambios en su forma de vestir o peinarse y que dos $(2-17,2 \%)$ de cada 10 hacen lo mismo con su pareja, respecto de estos cambios en la apariencia física de su novio o novia.

Entre dos (2 - 20,6\%) jóvenes de cada 10 participantes reconocen que su pareja lo hace sentir que no vale para nada y menos de dos (2 $-15,3 \%$ ) de cada 10 hacen lo mismo con su novio o novia.

Entre dos (2 -18,6\%) jóvenes de cada 10 participantes reconocen que su pareja lo culpa de la violencia que se sufre en el noviazgo y entre uno (1 - 13,3\%) de cada 10 participantes culpa a su novio o novia de la violencia en el noviazgo juvenil.

Resumiendo los datos anteriores, se muestra que el 31,3\% de los participantes expresaron haber padecido de su pareja algún tipo de violencia psicológica y humillación, y el 25,3\% reconoció que este tipo de violencia se la infligió a su pareja; además, si se promedian estos tipos de violencia psicológica (humillación) pasiva y activa se obtiene un promedio del $28,3 \%$, lo cual la ubica en la clasificación propuesta en la intensidad media con tendencia a la alta, tal como lo corrobora el estudio de Rivera, Allen, Rodríguez, Chávez y Lazcano (2007).

\section{Violencia psicológica (celos, control)}

La categoría cinco mostro una frecuencia más alta y se comportó de mayor a menor intensidad de incidentes presentados así: su pareja quiere saber en todo momento donde está/quiero saber en todo momento donde está mi pareja; su pareja intento ponerlo/a celoso/ intento ponerlo/a celoso a mi pareja; su pareja se pone celoso cuando le llaman al teléfono/se pone celoso cuando llaman al teléfono a su pareja; su pareja lo acusó de coquetear con las personas que hablaba/ ella o él acusó a su pareja de coquetear con las personas que hablaba; su pareja revisa sus objetos sin permiso/revisa los objetos de su pareja sin permiso; su pareja intentó vigilar llamadas, sin permiso/ intentó vigilar llamadas, sin permiso de su pareja. 
Cada seis $(6-60,3 \%)$ de 10 participantes reconocen que su pareja quiere saber en todo momento donde está y cinco $(5-52,5 \%)$ de cada 10 participantes señalan que han querido saber en todo momento donde está su novio o novia.

Seis (6 - 61\%) de cada 10 participantes reconocen que su pareja intento ponerlo/a celoso(a) y cinco $(5-51,6 \%)$ de cada 10 participantes señalan que han querido poner celoso(a) a su novio/a en la relación de noviazgo.

Entre 5 y $6(56,2 \%)$ jóvenes de cada 10 participantes reconoce que su pareja se pone celoso cuando le llaman al teléfono y con una cifra casi igual entre 5 y $6(55,6 \%)$ de los participantes señalan que se han puestos celosos con su novio o novia por este hecho.

Cinco (5 - 47\%) de cada 10 participantes reconocen que su pareja lo acusó de coquetear con las personas que hablaba y cuatro (40 \%) de cada 10 de los participantes señalan que lo han hecho en la relación de noviazgo.

Un poco más de cuatro (4 - 45,3\%) jóvenes de cada 10 participantes reconocen que su pareja revisa sus objetos sin permiso y un tanto menor de cuatro (4 - 42,9\%) de cada 10 participantes señalan que han revisado los objetos personales de su novio o novia.

Tres $(3-35,8 \%)$ de cada 10 participantes reconocen que su pareja intento vigilar llamadas sin permiso y con una cifra un poco más baja de tres $(3-34,9 \%)$ de cada 10 jóvenes, participantes señalan que lo han hecho en la relación de noviazgo.

Haciendo el ejercicio de promediar las tendencias en la violencia psicológica (celos, control), se encuentra que el 50,9\% reconoció haber recibido de su pareja este tipo de violencia psicológica en alguna de sus formas, así mismo, reconoció que la ocasionó a su pareja en el 46,2\% de los casos, lo que da un promedio total de $48,5 \%$ a esta categoría, convirtiéndola en el mayor tipo de violencia en el noviazgo juvenil en la población de jóvenes del Valle del Aburrá, participantes del estudio, y la cual puede clasificarse como alta (Jaramillo, Holguín y Ramírez, 2015). 
Si bien en esta investigación los jóvenes hacen un reconocimiento de que si hay diversas formas de violencia en el noviazgo juvenil, los presentes investigadores encontraron que la violencia psicológica (celos, control) es la más alta y humillación es media, siendo estas ultima la más recibida y ejercida entre ellos. También reconocen así mismo que las de menores frecuencias son la violencia física (baja) y sexual (la más baja de todas) (Jaramillo, Holguín y Ramírez, 2015).

Las cifras anteriores se pueden relacionar con el estudio de Peña, Zamorano, Hernández, Hernández, Vargas y Parra (2013) en el que indagaron a 140 jóvenes en Tamaulipas, México, sobre violencia en el noviazgo y las cifras que estos revelaron oscilaban entre "el 45,5\% y el 46,8\% de hombres y mujeres que han sufrido un acto de violencia" (p.26), este rango está muy cercano al de 48,5\% que reveló la violencia psicológica (celos, control) recibida y ejercida en las y los jóvenes del Valle de Aburrá, Colombia (Jaramillo, Holguín y Ramírez, 2015).

Los resultados cuantitativos de esta investigación apuntan a que los jóvenes evidencian que existen actos que parecieran ser simples formas de agresión física pero tienen intención de daño, y desde un análisis profundo deben ser considerados como actos violentos de carácter intencional. Si bien, no puede establecerse una relación causal y significativa entre género y violencia, pues según las investigaciones el 97,5\% de los varones y el 93,2\% de las mujeres, han identificado al menos un indicador de violencia en su relación de noviazgo. Si se tienen en cuenta las respuestas según los varones y según las mujeres, los resultados cobran importancia al analizar cada tipo de violencia en particular (Peña et al., 2013).

Además de las consideraciones sobre sí estas formas de violencia se presentaron en el último año o la violencia fue a lo largo de la vida, como es el caso de los datos que se presentan en la actual investigación, cabe anotarse que con todas esta variables difíciles de controlar, los datos se afectan de manera significativa, más si a ellas le sumamos: el tipo de población estudiada, el nivel socio-económico, los niveles de alfabetismo o analfabetismo por mencionar algunos importantes; ello se puede corroborar en investigacio- 
nes clásicas como las de Price, Byers, Sears, Whelan \& Saint-Pierre (2000); Grumbaum, Kann, Kinchen, Williams, Ross, Lowry \& Kolbel, (2002); Glass, Freland, Campbell, Yonas, Sharp \& Kub (2003) y actualizadas como las de: Urbiola, Estévez y Aguilera (2011); Urbiola, Estévez, Aguilera y Londoño (2011); Urbiola, Estévez y Iraurgi (2011a, 2001b), Urbiola, Estévez y Iraurgi (2014); Urbiola (2014) ; Jaramillo, Holguín y Ramírez (2015).

\section{Conclusiones}

En cuanto al reconocimiento del nivel de intensidad de la violencia en el noviazgo juvenil la cifra se inclina más hacia medio, entre dos y tres participantes de cada cinco, reconoce un nivel medio de violencia en el noviazgo juvenil, seguido de dos de cada cinco participantes que considera que es alto.

De acuerdo a este ejercicio investigativo, se puede afirmar que sí se visibiliza el problema de la violencia por nueve (9) de cada diez (10) jóvenes participantes en el estudio, los cuales consideran que su intensidad esta entre media y alta, para estas y estos la violencia de mayor impacto es la psicológica seguida de la física, esta última con un baja incidencia en la población indagada.

Los datos cuantitativos de esta investigación, evidencian que los jóvenes reconocen que la violencia predominante es la violencia psicológica (Blázquez, Moreno y García, 2011), expresada a través los celos, el control y de la humillación, estas son las formas de violencia más recibidas y ejercidas entre ellos, y la de menor recurrencia es la violencia física.

En cuanto a la violencia física los jóvenes se muestran de acuerdo que en la relación de noviazgo, no se percibe la presencia predominante de las agresiones físicas, parece que las representan socialmente como parte de su experiencia cotidiana en los otros jóvenes o adultos que conforman familias, con los que se relacionan, mas no se percibe con una alta incidencia en los entrevistados, y muchas veces no las nombran o para estos pasan desapercibidas o son silenciadas. 
Dentro de los actos más frecuentes de humillación en las relaciones de pareja juvenil, las y los jóvenes testimonian haber recibido por parte de su pareja un insulto cuando su novio(a), estaba enojado con ella o él, o con otras personas, y consideran que es la forma de violencia psicológica más recurrente, pues dos jóvenes de cada uno de los participantes reportó haber vivido esta experiencia.

En cuanto a lo preguntado en referencia a la violencia sexual, la mayoría de los jóvenes afirma nunca haberla padecido. Solo una mínima parte de la población participante, hablo de haber padecido este tipo de violencia, en los casos que se presentó, fue una figura masculina y se relacionó con las actitudes machistas y de ejercicio de poder sobre el cuerpo y la vida del otro.

Las violencias psicológicas son un componente muy significativo y reconocido en Colombia, pero lo que se nombra en esta investigación llama la atención pues en los análisis cuantitativos se encontró que no son altos en los ítems evaluados por el V.E.R.A., aquí es pertinente preguntarse si los jóvenes realmente tienen relaciones sin violencia $u$ ocultaron información de cara a sus relaciones de pareja, o si es que no reconocen que existen otros tipos violencias que son socialmente vistas por los adultos pero que no son nombradas, reconocidas o visualizadas por las y los jóvenes.

\section{Referencias}

Alvarado, S., Martínez, J. E., y Gaviria, D. (2009). Contextualización teórica al tema de las juventudes: una mirada desde las ciencias sociales a la juventud Revista latinoamericana de ciencias sociales, niñez y juventud, 7(1), 83-102. Recuperado de: http://redalyc.uaemex.mx/src/inicio/ArtPdfRed.jsp?iCve=7730710. Amurrio, M., Larrinaga, A., Usategui, E., y Del Valle, A. (2008). Violencia de género en las relaciones de pareja de adolescentes y jóvenes de Bilbao. Bilbao, España: Área de Igualdad, Cooperación y Ciudadanía Ayuntamiento de Bilbao. 
Araya, S. (2002). Las representaciones sociales. Ejes para su discusión. Costa Rica: Facultad Latinoamericana de Ciencias Sociales (Flacso). Recuperado en: http://www.flacso.or.cr/fileadmin/documentos/FLACSO/Cuaderno127.pdf.

Ariza, G. (2009). Las representaciones sociales de la violencia en las relaciones de pareja en la prensa de Medellín en el Siglo xxı: El colombiano 2001 - 2008. La chiva 2002-2008. Revista Venezolana de Estudios de la Mujer, Caracas, 14(32), 71-97. Recuperado de http://www.scielo.org.ve/scielo.php?script=sci_arttext\&pid=S1316-37012009000100008\&lng=es\&nrm=iso.

Ariza, G. R. (2013). Las representaciones sociales de la violencia en las relaciones de pareja en Medellín en el Siglo xxi. CES Psicol [online], 6(1), 134-158. Recuperado de http://www.scielo.org.co/scielo.php?script=sci_arttext\&pi$\mathrm{d}=$ S2011-30802013000100009.

Blázquez, M., Moreno, J., y García, M. (2011). Desarrollo de la violencia psicológica durante el noviazgo en parejas de jóvenes universitarios(as). Apuntes de Psicología, 29(3), 397-412. Recuperado de http://dialnet.unirioja.es/servlet/ articulo?codigo $=4026866$.

Botero, W., Herrera, H. D., Reyes, J. I., y Urrea, F. (2006). Afecto y elección de pareja en jóvenes de sectores populares de Cali. Revista Estudios Feministas. 14(1). 117-148. Recuperado de http://www.redalyc.org/articulo.oa?id=38114108.

Caycedo E.C., Cubides, I., Martín, A., Cortés, O. F., Berman, S., Oviedo, A. M., y Suárez, I. (2007). Relación entre el género y las experiencias de cortejo y actitudes hacia las relaciones románticas en adolescentes bogotanos. Psicología desde el Caribe, (20), 76-92. Recuperado de http://www.redalyc.org/articulo. oa?id=21302005.

Centers for Disease Control and Prevention (CDC, 2013). Understanding teen dating violence. Recuperado de http://www.cdc.gov/violenceprevention/intimatepartnerviolence/teen_dating_violence.html 
Congreso de Colombia (2013). Estatuto de Ciudadanía Juvenil Ley 1622. Bogotá, Colombia: Congreso de la República. Recuperado de: http://www.mininterior. gov.co/sites/files/ley_1622_del_29_de_abril_de_2013.

Cortés M, Bringas, M., Rodríguez, F., Flores G, Sánchez, R., y Rodríguez, J (2014). Unperceived dating violence among Mexican students. International Journal of Clinical and Health Psychology, 14, 39-47. Recuperado de http://www. elsevier.es/es-revista-international-journal-of-clinical-and-355-articulo-unperceived-dating-violence-among-mexican-90260036.

Dirección Seccional de Salud y Protección Social de Antioquia. (2010). Boletín epidemiológico Salud Mental. Medellín, Colombia: Dirección Seccional de Salud y Protección Social de Antioquia.

Downey, G., Bonica, C., y Rincón, C. (1999). Rejection Sensitivity And Adolescent Romantic Relationships. En W. Furman, C. Feiring \& B.B Brown, (Eds.). The development of romantic relationships in adolescence (pp.148-174). London: Cambridge University Press, Recuperado de http://catdir.loc.gov/catdir/samples/camo32/98032339.pdf.

Duschatzky, S., y Corea, C. (2009). Chicos en banda: los caminos de la subjetividad en el declive de las instituciones: Buenos Aires: Paidós.Echeburúa, E., Fernández, J., y Corral, P. (2008). ¿Hay diferencias entre la violencia grave y la violencia menos grave contra la pareja?: un análisis comparativo. International Journal of Clinical and Health Psychology, 8(2), 355-382. Recuperado de http://www.redalyc.org/articulo.oa?id=33712001001.

Escoto, Y., González, M., Muñoz, A., y Salomon, Y. (2007, julio). Violencias en el noviazgo adolescente. Revista internacional de psicología. Recuperado de http://psicologiarevista.99k.org/Violencia\%20en\%20el\%2onoviazgo\%20adolescente.pdf.

Estévez, A., Ocerinjauregui, N., Escudero, C., Urbiola, I., y Redondo, I. (16-19 de marzo, 2011). Psychological Effects on Women of Sexual Abuse in Childhood. $4^{\text {th }}$ World Congress on Women's Mental Health. Madrid, España. 
Estévez, A., Ocerinjauregui, N., Escudero, C., Urbiola, I., Redondo, I., y Londoño, N. H. (26 - 30 de junio, 2011). Consecuencias psicológicas del abuso sexual en la infancia en la mujer. Ponencia, XXXIII Congreso Interamericano de Psicología, Medellín, Colombia. Ferrer Pérez, V. A., Bosch Fiol, E., Ramis Palmer, M. C., y Navarro Guzmán, C. (2006). Las creencias y actitudes sobre la violencia contra las mujeres en la pareja: Determinantes sociodemográficos, familiares y formativos. Anales de Psicología, 22(2), 251-259. Recuperado de http://www. redalyc.org/articulo.oa?id=16722210.

Galeano, E. (2009). Diseño de Proyectos en la Investigación Cualitativa. Medellin: Universidad Eafit, Quinta reimpresión.

Glass, N., Fredland, N., Campbell, J., Yonas, M., Sharps, P., Kub, J. (2003). Adolescent dating violence: Prevalence, risk factors, health outcomes, and implications for clinical practice. Journal of obstetric, gynecologic, and neonatal nursing, 32(2), 227-237. Recuperado de http://www.ncbi.nlm.nih.gov/pubmed/12685675.

Gobernación de Antioquia. (2013). Eventos de interés en salud pública por subregiones y municipios. Antioquia 2007 - 2012. Dirección Seccional de Salud de Antioquia. Recuperado de http://www.dssa.gov.co/__media_/dssa/dssa. gov.co/images/stories/documentos/eventos_salud_publica_por_municipio_2007_2012.xls.

Gobierno de Bilbao (2008). Violencia de género en las relaciones de pareja de adolescentes y jóvenes de Bilbao. Conclusiones de informe. Bilbao, España. Recuperado de http://docplayer.es/14322220-Conclusiones-del-informe-violencia-de-genero-en-las-relaciones-de-pareja-de-adolescentes-y-jovenes-de-bilbao.html.

González, R., y Santana, J. D. (2001). La violencia en parejas jóvenes. Psicothema. 13(1), 127-131. Recuperado de http://www.redalyc.org/articulo.oa?id=72713118. 
Graves, K. N., Sechrist, S. M., White, J. W., \& Paradise, M. J. (2005). Intimate partner violence perpetrated by college women within the context of a history of victimization. Psychology of women quarterly, 29(3). 278-289. doi: 10.1111/j.1471-6402.2005.00222.x.

Gray, M. R., \& Steinberg, L. (1999). Adolescent romance and the parent-child relationship: A contextual perspective. En W. Furman, B. B. Brown \& C. Feiring (Eds.). The development of romantic relationships in adolescence (pp. 235262). London: Cambridge University Press, Recuperado de http://catdir.loc. gov/catdir/samples/camo32/98032339.pdf.

Hernández, R., Fernández, C., y Baptista, P. (2006). Metodología de la Investigación. Mexico: McGawHill. Cuarta edición.

Jaramillo, C., Holguín, H., y Ramírez, L. (2015). Algunas representaciones sociales sobre la violencia en los noviazgos juveniles heterosexuales en los municipios del Valle de Aburrá. Medellín: Editorial IUE y L.Vieco e Hijas Ltda.

Jodelet, D. (1985). Representaciones sociales. En: Moscovici. Psicología social 1. Buenos Aires, Argentina: Paidós.

Maldonado, M. M. (2005). Noviazgo, emotividad y conflicto. Relaciones sociales entre alumnos de la escuela media Argentina. Revista Mexicana de Investigación Educativa. 1O(26), 719-737. Recuperado de http://redalyc.org/articulo. oa?id=14002606.

Margullis, M., y Urresti, M. (2008). La juventud es más que palabras. Ed. Biblos Sociedad. Recuperado de http://perio.unlp.edu.ar/teorias/index_archivos/ margulis_la_juventud.pdf.

Martínez, I., Bonilla, A., Gómez, L., y Bayot, A. (2008). Identidad de género y afectividad en la adolescencia: asimetrías relacionales y violencia simbólica. Anuario de Psicología. 39(1), 109-118. Recuperado de http://www.redalyc.org/articulo. oa?id=97017401010. 
Martínez, L. W. (2003). La violencia de género en la relación de pareja ¿una cuestión no perceptible en la vida cotidiana? Revista de Ciencias Sociales. (Ve), Ix(2), 235-251. Recuperado de http://www.redalyc.org/articulo.oa?id=28009205.

Mingo, A. (2010). Ojos que no ven... Violencia escolar y género. Perfiles educativos. xxxII(130), 25-48. Recuperado de http://www.scielo.org.mx/pdf/peredu/ v32n13o/v32n130a3.pdf.

Moscovici, S. (1985). Psicología Social I. Buenos Aires: Ediciones Paidós.

Nullvalue, (octubre 11 de 2010). Violencia de pareja se inicia a los 11 años. El Tiempo. Recuperado de http://www.eltiempo.com/archivo/documento/MAM4185314 .

Organización Panamericana de la Salud, Organización Mundial de la Salud (2011). Prevención de la violencia sexual y violencia infligida por la pareja contra las mujeres: Qué hacer y cómo obtener evidencias. Washington, DC: Organización Panamericana de la Salud. Recuperado de http://apps.who.int/iris/ bitstream/10665/44810/1/9789275316351_spa.pdf.

Ortiz, O., Barrera, D., y Ramírez, L. (2003). Unidad Introductoria. En: Prevención Temprana de la agresión. Orientaciones para el docente en el aula. Medellín, Colombia: Edúcame.

Penagos, A., Rodríguez, M., Carrillo, S., y Castro, J. (2006). Apego, relaciones románticas y autoconcepto en adolescentes bogotanos. Universitas Psychologica, 5(1) 21-36. Recuperado de http://www.redalyc.org/articulo.oa?id=64750103.

Peña, F., Zamorano, B., Hernández, G., Hernández, M., Vargas, J., y Parra, V. (2013) Violencia en el noviazgo en una muestra de jóvenes mexicanos. Revista costarricense de psicología, 32(1), 27-40. Recuperado de https://dialnet.unirioja. es/descarga/articulo/4836509.pdf.

Póo, A. M., y Vizcarra, M. B. (2008). Violencia de Pareja en Jóvenes Universitarios. Terapia psicológica, 26(1), 81-88. Recuperado de http://www.scielo.cl/scielo. php?script=sci_arttext\&pid=So718-48082008000100007\&lng=es\&tlng=es. 10.4067/So718-48082008000100007. 
Price, L., Byers, S., Sears, H., Whelan, J., \& Saint, M. (2000). Dating Violenceamongst New Brunswick Adolescents: A summary of two studies. Research Paper: University of New Brunswick, Muriel McQueen Fergusson Centre for Family Violence Research. Recuperado de http://www.unb.ca/fredericton/ arts/centres/mmfc/_resources/pdfs/datingviolence.pdf.

Programa integral sobre las violencias de género (2010). Estudio sobre tolerancia social e institucional a la violencia basada en género en Colombia. Unifem, Unfpa y Oim. Recuperado de http://www.mdgfund.org/sites/default/files/ gen_estudio_colombia_tolerancia\%20social\%20e\%20institucional\%20a\%20 la\%2oviolencia\%2ode\%2ogenero.pdf.

Ramírez, C., y Núñez, D. (2010). Violencia en la relación de noviazgo en jóvenes universitarios: un estudio exploratorio. Enseñanza e Investigación en Psicología, 15(2), 273-283. Xalapa. Recuperado de http://www.redalyc.org/ pdf/292/29215980003.pdf.

Rey-Anacona, C. A. (2009). Maltrato de tipo físico, psicológico, emocional, sexual y económico en el noviazgo: Un estudio exploratorio. Acta Colombiana de Psicología, 12(2), 27-36. Recuperado de http://www.redalyc.org/articulo. oa?id=79815640003.

Rey-Anacona, C. A. (2008). Prevalencia, factores de riesgo y problemáticas asociadas con la violencia en el noviazgo: una revisión de la literatura. Avances en Psicología Latinoamericana, 26(2), 227-241. Recuperado de http://www.redalyc. org/articulo.oa?id=79926209.

Rey-Anacona, C. A., Mateus-Cubides, A. M., y Bayona-Arévalo, P. A. (2010). Malos tratos ejercidos por adolescentes durante el noviazgo: diferencias por sexo. Revista Mexicana de Psicología, 27(2), 169-181. Recuperado de http://www. redalyc.org/articulo.oa?id=243016324006.

Rivera, L., Allen, B., Rodríguez, G., Chávez, R., y Lazcano, E. (2006). Violencia durante el noviazgo, depresión y conductas de riesgo en estudiantes femeninas (1224 años). Salud Pública de México, 48(2), 288-296. Recuperado de http:// www.redalyc.org/articulo.oa?id=10604809. 
Rivera, L., Allen, B., Rodríguez, G., Chávez, R., y Lazcano, E. (2007). Prevalence and correlates of adolescent dating violence: Baseline study of a cohort of 7960 male and female Mexican public school students. Preventive Medicine, 44(6), 477-484. Recuperado de http://www.ncbi.nlm.nih.gov/pubmed/17467784.

Rodríguez, I. (2013). La dependencia emocional en las relaciones interpersonales. ReiDoCrea. Revista electrónica de investigación Docencia Creativa, 2, 143148. Recuperado de http://digibug.ugr.es/bitstream/10481/27754/1/ReiDoCrea-Vol.2-Art.19-Rodriguez.pdf.

Rodríguez, L. (2012). Subjetividades, adolescencias y violencias. Tesis Psicológica, 7, 188-193. Recuperado de http://www.redalyc.org/articulo. oa?id=139025258015.

Rodríguez, V. Sánchez, C., González, D. (2006). Creencias de adolescentes y jóvenes en torno a la violencia de género y las relaciones de pareja. Portularia, vI(2), 189-204. Recuperado de http://www.redalyc.org/articulo. oa?id=161017317010.

Rojas, F., Muñoz, G., y Corredor, L.C. (2012). Jóvenes y adultos: una pedagogía del encuentro. Bogotá: Pontificia Universidad Javeriana. Recuperado de http:// ceanj.cinde.org.co/wp-content/uploads/2013/o2/Jovenes-y-adultos.pdf.

Rojas-Solís, J. (2013). Violencia en el noviazgo de adolescentes mexicanos: Una revisión. Revista de educación y desarrollo, 27, 49-58. Recuperado de http:// www.cucs.udg.mx/revistas/edu_desarrollo/anteriores/27/o27_Rojas.pdf.

Romero, A., Pick, S., Parra Coria, A., y Givaudan, M. (2010). Evaluación del Impacto de un Programa de Prevención de Violencia en Adolescentes. Interamerican Journal of Psychology. 44(2) 203-212. Recuperado de http://www.redalyc. org/articulo.oa?id=28420641001.

Rubio-Garay, F., López-González, M. A., Saúl, L. A., Sánchez-Elvira-Paniagua, A. (2012). Direccionalidad y expresión de la violencia en las relaciones de noviazgo de los jóvenes. Acción Psicológica, 9(1), 61-70. doi:http://dx.doi. org/10.5944/ap.9.1.437. 
Salomón, Y., Escoto, Y., González, M., y Muñoz, A. (2007). Violencia en el noviazgo adolescente. Revista internacional de Psicología. 8(02), 1-34. Recuperado de http://psicologiarevista.99k.org/Violencia\%20en\%20el\%2onoviazgo\%20 adolescente.pdf.

Samaniego, E., y Fleixar, A. (2010). Estudio sobre la identificación y vivencia de violencia en parejas adolescentes. Apuntes de Psicología, 28(3), 349-366. Recuperado de http://dialnet.unirioja.es/servlet/articulo?codigo=3733211.

Sánchez, L., Gutiérrez, M.E., Ballesteros, M., Izzedin, R., y Gómez, A. (2011). Representaciones sociales del noviazgo, en adolescentes escolarizados de estratos bajo, medio y alto, en Bogotá. Recuperado de http://redalyc.uaemex.mx/src/ inicio/ArtPdfRed.jsp?iCve $=42219906007$.

Secretaría de Equidad y de Género para las Mujeres (s.f.). Lecciones para la equidad. Guía de coeducación. Manuales de autoaprendizaje. Por una educación no sexista. Gobernación de Antioquia. Medellín, Colombia: Gobernación de Antioquia.

Urbiola, I. (2014). Violencia recibida, ejercida y percibida en las relaciones de noviazgo de jóvenes y adolescentes. Tesis Doctoral. Universidad de Deusto, Bilbao.

Urbiola, I., Estévez, A., \& Aguilera, L. (2011). A prevention programme on violence against women in adolescent dating violence. 4th World Congress on Women’s Mental Health. Madrid, España.16-19 de Marzo. Recuperado de http:// www2.kenes.com/iawmh_es/pages/home.aspx.

Urbiola, I., Estévez, A., Aguilera, L., y Londoño, N. (26-30 de junio, 2011). Resultados preliminares de un programa preventivo de la violencia en las relaciones de noviazgo adolescente. III Congreso Interamericano de Psicología. Medellín, Colombia.Urbiola, I., Estévez, A., y Iraurgi, I. (7, 8 y 9 de julio, 2011). Dependencia emocional en jóvenes y adolescentes: resultados preliminares de la Escala de Dependencia. Ix Congreso nacional de psicología clínica, San Sebastián, España. Recuperado de http://www.ugr.es/ aepc/WEBCLINICA/ DOCUMENTOS/libroresumenesIXcongreso.pdf. 
Urbiola, I., Estévez, A., y Iraurgi, I. (7, 8 y 9 de Julio, 2011) . Evaluación de la violencia recibida y ejercida en las parejas adolescentes y jóvenes. Ix Congreso Nacional de Psicología Clínica, San Sebastián, España. Invitada simposium violencia en jóvenes y adolescentes. Simposium Violencia en jóvenes y adolescentes. Recuperado de http://www.ugr.es/ aepc/WEBCLINICA/DOCUMENTOS/libroresumenesIXcongreso.pdf.

Urbiola, I., Estévez, A., y Iraurgi, I. (2014). Violencia sexual psicológica y percepción de la violencia en adolescentes y jóvenes según el cuestionario V.E.R.A. Inguruak Revista Vasca de Sociología y Ciencia Política, 56-57, 1424-1433. Recuperado de http://www.civersity.net/files/55-56.pdf.

Vargas, E., y Barrera, F. (2002). Adolescencia, relaciones románticas y actividad sexual: Una revisión. Revista Colombiana de Psicología, (11), 115-134. Recuperado de http://www.revistas.unal.edu.co/index.php/psicologia/article/ viewFile/1203/1753.

Vázquez García, V., y Castro, R. (2008). “'Mi novio sería capaz de matarme?” Violencia en el noviazgo entre adolescentes de la Universidad Autónoma Chapingo, México. Revista Latinoamericana de Ciencias Sociales, Niñez y Juventud, 6(2) 709-738. Recuperado de http://www.redalyc.org/articulo. oa?id=77360208.

Velázquez R. G. (2011). La violencia durante el noviazgo. Elementos: Ciencia y Cultura, 19(82), 39-43. Recuperado de http://www.redalyc.org/ pdf/294/29418148007.pdf.

Villaseñor, M., y Castañeda, J. D. (2003). Masculinidad, sexualidad, poder y violencia: análisis de significados en adolescentes. Salud Pública de México. 45(1) 44-57. Recuperado de http://www.redalyc.org/articulo.oa?id=10609508.

Wolfe, D. A., Scott, K., Reitzel-Jaffe, D., Wekerle, C., Grasley, C., \& Straatman, A. (2001). Development and validation of the conflict in Adolescent Dating Relationships Inventory. Psychological Assessment, 13(2), 277-293. doi:10.1037//1040-3590.13.2.277. 